\title{
Causal Entropy-Complexity Plane with Multivariate Probability Distribution ${ }^{\dagger}$
}

\section{Givanildo Nascimento, Jr ${ }^{1}$, Cristopher Freitas ${ }^{1}$, Osvaldo Rosso ${ }^{2,3}$ and André Aquino ${ }^{1}$}

1 Instituto de Computação, Universidade Federal de Alagoas, Maceió, AL, Brazil

2 Instituto de Física, Universidade Federal de Alagoas, Maceió, AL, Brazil

3 Instituto de Medicina Traslacional e Ingeniería Biomedica, Hospital Italiano de Buenos Aires \& CONICET, Ciudad Autónoma de Buenos Aires, Argentina

+ Presented at the Entropy 2021: The Scientific Tool of the 21st Century, 5-7 May 2021; Available online: https://sciforum.net/conference/Entropy2021/.

Published: 5 May 2021

We propose a multivariate causality entropy-complexity plane by using a multivariate probability distribution function (PDF) and adapting the Normalized Permutation Entropy $\mathrm{H}$ and Statistical Complexity C.

Alongside the standard embedding dimension $\mathrm{D}$ for ordinal patterns of length $\mathrm{D}$ !, we considered sub-patterns of embedding dimensions that get more information in the phase space, since the order patterns do not always detect sensory changes in the time series. This level of complexity can capture details of the probability distribution of the system that are not discriminated by measures of randomness, such as entropy, thus the multivariate causality entropy-complexity plan is adopted.

In this application, the plane was used to analyze complex time series and the results indicate robustness in the distinction between chaotic systems, even after the insertion of noise in each of the chaotic time series that defines the system. Thus, the multivariate plan is more objective to extract the structure of a system and, thus, to characterize it.

(C) 2021 by the authors. Licensee MDPI, Basel, Switzerland. This article is an open access article distributed under the terms and conditions of the Creative Commons Attribution (CC BY) license (http://creativecommons.org/licenses/by/4.0/). 